\title{
Interaction of Molecular and Atomic Hydrogen With Single-Wall Carbon Nanotubes
}

\author{
Julio A. Alonso, Juan S. Arellano, Luis M. Molina, Angel Rubio, and María J. López
}

\begin{abstract}
Density functional calculations are performed to study the interaction of molecular and atomic hydrogen with $(5,5)$ and $(6,6)$ single-wall carbon nanotubes. Molecular physisorption is predicted to be the most stable adsorption state, with the molecule at equilibrium at a distance of 5-6 a.u. from the nanotube wall. The physisorption energies outside the nanotobes are approximately $0.07 \mathrm{eV}$, and larger inside, reaching a value of $0.17 \mathrm{eV}$ inside the $(5,5)$ nanotube. Although these binding energies appear to be lower than the values required for an efficient adsorption/desorption operation at room temperature and normal pressures, the expectations are better for operation at lower temperatures and higher pressures, as found in many experimental studies. A chemisorption state with the molecule dissociated has also been found, with the $\mathrm{H}$ atoms much closer to the nanotube wall. However, this state is separated from the physisorption state by an activation barrier of $2 \mathrm{eV}$ or more. The dissociative chemisorption weakens carbon-carbon bonds, and the concerted effect of many incoming molecules with sufficient kinetic energies can lead to the scission of the nanotube.
\end{abstract}

Index Terms-Carbon nanotubes, graphite, hydrogen absorption.

\section{INTRODUCTION}

$\mathbf{T}$ HE adsorption of hydrogen by graphitic materials, including carbon nanotubes, has been investigated by several groups, but the reports on the adsorption capacity are controversial [1]-[5]. Additional experiments on well-controlled samples are required to resolve the discrepancies. Also, theoretical calculations have the potential of being very useful to understand the nature of the adsorption and storage processes [6]. Computer simulations of hydrogen adsorption have been performed using effective model potentials to describe the

Manuscript received June 12, 2003; revised January 18, 2004. This work was supported by the Ministerio de Ciencia y Tecnología (MCYT) of Spain under Grant MAT2002-04 499-C02-01, by Junta de Castilla y León under Grant CO01/102, and by the European Community (RTN-COMELCAN). The work of J. A. Alonso was supported by the Donostia International Physics Center, San Sebastián, Spain. The work of M. J. López was supported by the MCYT of Spain under the Ramón y Cajal Program. An earlier version of this paper was presented at the Symposium of Microtechnologies for the New Millennium 2003, Nanotechnology Conference.

J. A. Alonso is with the Departamento de Física Teórica, Universidad de Valladolid, 47011 Valladolid, Spain and also with the Donostia International Physics Center, 20018 San Sebastián, Spain (e-mail: jaalonso@fta.uva.es).

J. S. Arellano is with the Area de Física Atómica Molecular Aplicada, Universidad Autónoma Metropolitana Azcapotzalco, 02200 México City, México.

L. M. Molina is with the Institute of Physics and Astronomy, Aarhus University, DK-800 Aarhus C, Denmark.

A. Rubio is with the Donostia International Physics Center, 20018 San Sebastián, Spain.

M. J. López is with the Departamento de Física Teórica, Universidad de Valladolid, 47011 Valladolid, Spain.

Digital Object Identifier 10.1109/TNANO.2004.828678 interactions, but only first principles electronic structure calculations can give an accurate description of the interaction of hydrogen with carbon nanotubes and other graphitic materials.

The interaction of atomic hydrogen with single-wall carbon nanotubes (SWNT) has been studied using the density functional theory (DFT), and Gülseren et al. [7], [9] and Yildirim et al. [8] found that the binding energy depends sensitively on the curvature of the nanotube. Although the chemisorption of atomic hydrogen is an important issue, the technologically relevant process appears to be the physisorption of molecular hydrogen. Early attempts of studying the adsorption of molecular hydrogen on the nanotube walls failed because the generalized gradient approximations (GGAs) employed to describe exchange and correlation effects between the electrons give a purely repulsive interaction between the closed shell molecule $\mathrm{H}_{2}$ and the nanotube wall [10]-[12], a prediction that is in disagreement with the experiments for adsorption on graphite pores, nanotube bundles, and nanofibers. We have found that the local density approximation (LDA) to exchange and correlation allows for an accurate unified treatment of the molecular and atomic adsorption of hydrogen that clarifies the relation between the two processes. Under normal conditions, molecular physisorption is preferred. However, if by energetic impact, or under high pressure, the $\mathrm{H}_{2}$ molecules can approach sufficiently close to the nanotube wall, the molecules can dissociate, and then atomic chemisorption becomes possible. The atomic chemisorption weakens substantially the carbon-carbon (C-C) bonds.

\section{COMPUTATIONAL MEthod}

The calculations of the interaction between the hydrogen atom, or the hydrogen molecule, and carbon nanotubes (or graphite) have been performed with the ab initio FHI96MD code [13]. This density functional code uses a periodic supercell geometry and a plane-wave expansion for the electronic wave functions. The $1 s^{2}$ cores of the carbon atoms are replaced by the nonlocal norm-conserving pseudopotentials of Bachelet $e t$ al. [14] and Hamman et al. [15]. Below, we present results for static calculations and dynamical simulations of the interaction of $\mathrm{H}$ and $\mathrm{H}_{2}$ with $(5,5)$ and $(6,6)$ nanotubes. The supercell contains 20 carbon atoms in the case of $(5,5)$ nanotubes, and 48 carbon atoms in the case of $(6,6)$ nanotubes. Several tests were performed to control the influence of the cell dimensions and the magnitude of the energy cutoff of the plane-wave basis. The results for nanotubes are complemented with the study of the interaction of $\mathrm{H}_{2}$ with a planar graphitic layer. 
TABLE I

BINDING ENERGIES (IN ELECTRONVOLTS) OF $\mathrm{H}_{2}$ TO GRAPHITE AND NANOTUBES

\begin{tabular}{l|l|l}
\hline & To graphite & To nanotubes \\
\hline This work (DFT) & 0.09 & 0.07 outside $(5,5)$ \\
\hline & & 0.17 inside $(5,5)$ \\
\hline & & 0.07 outside $(6,6)$ \\
\hline Okamoto (MP2) [18] & 0.09 & 0.12 inside (6,6) \\
\hline Li (DFT) [20] & & 0.11 inside (7,7) \\
\hline Mattera (exp) [21] & 0.04 & \\
\hline Brown (exp) [24] & & 0.06 (radius 13 a.u) \\
\hline Narehood (exp) [25] & & 0.02 \\
\hline
\end{tabular}

\section{PhySISORPTION OF $\mathrm{H}_{2}$ ON GRAPHITE AND CARBON NANOTUBES}

The interaction between $\mathrm{H}_{2}$ and a planar graphene layer has been studied in [16]. The equilibrium position is obtained for the molecule in a position above the center of a carbon hexagon, with the molecular axis parallel to the graphene plane. The distance from the center of mass of the molecule to the layer is 5.1 a.u. and the binding energy of the molecule to the graphene layer is $0.086 \mathrm{eV}$. The electron densities of a pure graphene layer in a plane 5.1 a.u. above the layer are in the range from $6 \times 10^{-5}$ e/a.u. ${ }^{3}$ to $9 \times 10^{-5}$ e/a.u. ${ }^{3}$ so the $\mathrm{H}_{2}$ molecule is in equilibrium in the tail of the graphene electron density. The main contribution to the binding comes from the exchange and correlation effects arising from the weak overlap between the electron densities of the graphene layer and the closed-shell $\mathrm{H}_{2}$ molecule. Even without any density rearrangement, exchange and correlation effects produce an attractive contribution [17]. In addition, there is a very small density rearrangement [16] that contributes to the weak bonding. All these features indicate a physisorption state. The weakly attractive $\mathrm{H}_{2}$-graphene interaction potential obtained by the LDA is in remarkable agreement with that calculated using Möller-Plesset perturbation theory (MP2) [18] (a comparison of the binding energies is provided in Table I). The simulation of the diffusion of the molecule from one hollow site to another in a neighbor hexagon, following the shortest path, predicts an activation energy $\Delta E_{a}=0.014 \mathrm{eV}(163 \mathrm{~K})$. This is in good agreement with the activation energy for the diffusion of deuterium hydride (HD) adsorbed on exfoliated graphite $\Delta E_{a}=152 \pm 20 \mathrm{~K}$, measured by Bienfait et al. [19] using quasi-elastic neutron scattering.

Li et al. [20] have performed a simple thermodynamic analysis of the adsorption/desorption process based on a comparison of the free energies of the gas and adsorbed phases of hydrogen. For the absorption/desorption from the surface of graphitic materials to be effective under conditions relevant for the requirements of the automotive industry, that is near room-temperature and normal pressures. Li et al. [20] have estimated that a

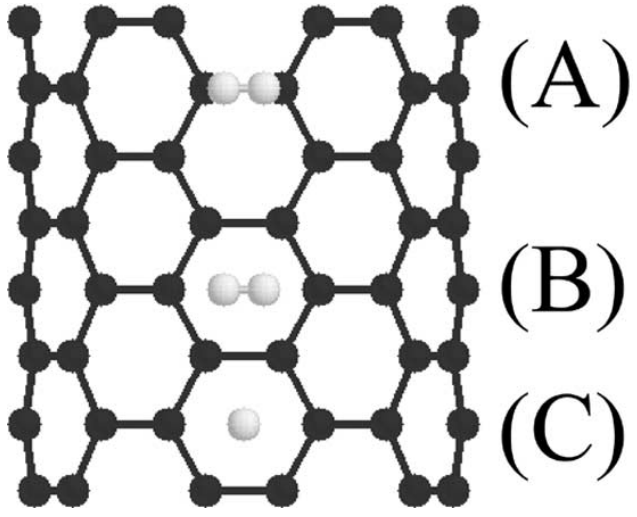

Fig. 1. Configurations of the $\mathrm{H}_{2}$ molecule above a $\mathrm{C}-\mathrm{C}$ bond (A), and above an hexagon with the molecular axis parallel (B) and perpendicular (C) to the nanotube wall.

binding energy of $0.15-0.2 \mathrm{eV}$ per $\mathrm{H}$ atom is required. However, the calculated binding energies of $\mathrm{H}_{2}$ to the graphene layer reported above are substantially smaller, and the same occurs with the experimental binding energies [21]. Evidently, the requirements would be less strict under conditions of cryogenic (liquid nitrogen) temperatures and, as discussed by Li et al. [20], the calculated $\mathrm{H}_{2}$-graphene binding energies [16] appear to explain some of the reports of adsorption of hydrogen by graphitic materials.

The question then arises if the curvature of the graphene layer, which is characteristic of carbon nanotubes, can increase the binding energy [20], [22], [23]. For this purpose, we have performed calculations of the adsorption of $\mathrm{H}_{2}$ by $(5,5)$ and $(6,6)$ carbon nanotubes. After optimization of the structure of a free $(5,5)$ SWNT, which gives an average $\mathrm{C}-\mathrm{C}$ bond length of 2.68 a.u. and a nanotube radius $R=6.44$ a.u., a hydrogen molecule was placed in different sites and orientations relative to the nanotube, as shown in Fig. 1, and the energy of the system was calculated as a function of the distance $D_{a}$ from the center of mass of the molecule to the nanotube axis. The bond length of the molecule was maintained fixed at 1.48 a.u., and the coordinates of all the carbon atoms of the nanotube were kept frozen. From experience with the planar graphene layer [16], we know that these assumptions are valid in the region of the physisorption minimum. Fig. 2 shows that weak physisorption wells are obtained outside and inside the nanotube, and that the absolute energy minimum occurs for the molecule at the center of the nanotube. The binding energies outside (for the most stable configuration) and inside are 0.068 and $0.17 \mathrm{eV}$, respectively, and the difference reflects the opposite curvature seen by the hydrogen molecule inside and outside the nanotube. Inside the nanotube, the molecule is effectively sampling a larger nanotube area. The binding energy for the case of the planar graphene layer, i.e., $0.086 \mathrm{eV}$, is, in fact, intermediate between those two values. For the same reason, the short-range repulsion between the molecule and SWNT becomes stronger inside the nanotube. A comparison of the different curves for $\mathrm{H}_{2}$ outside the nanotube indicates that physisorption on a hollow position is preferred over other sites, which is the same as for graphene. 


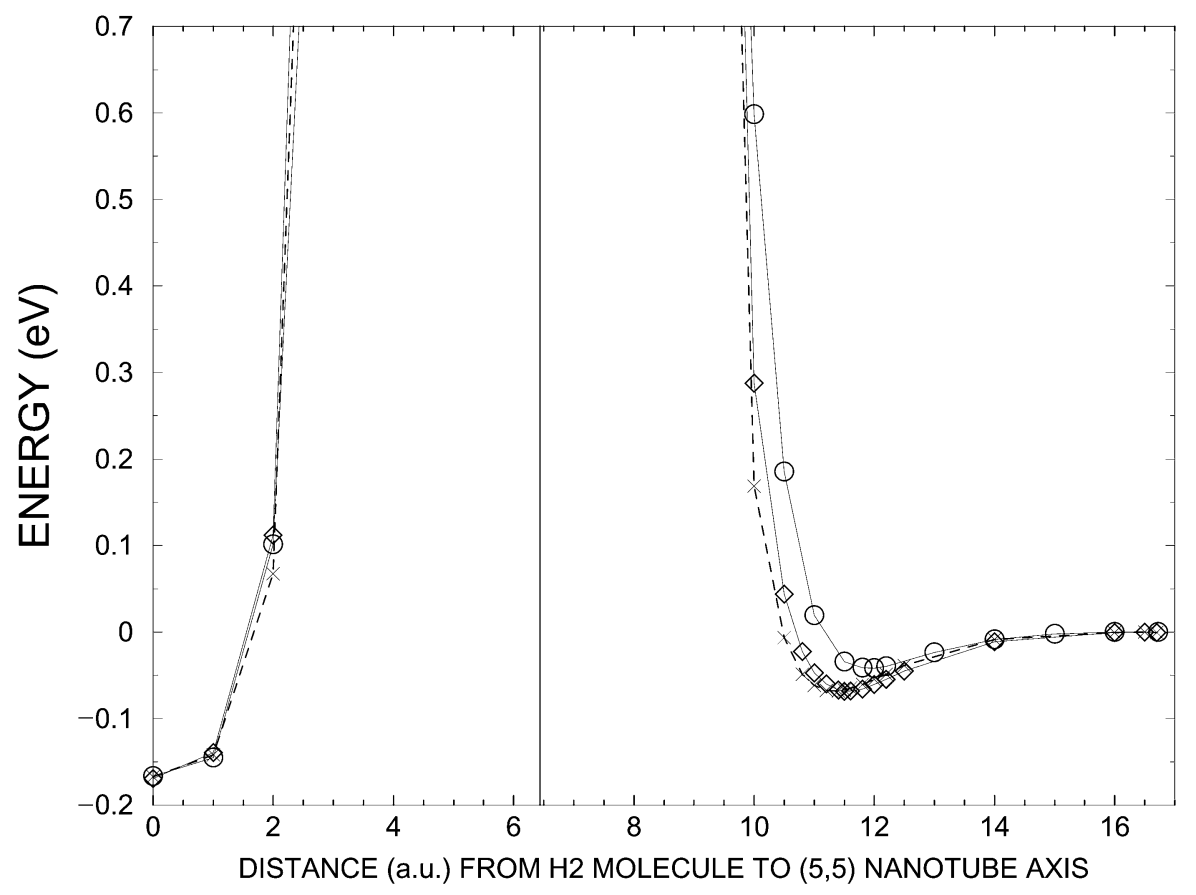

Fig. 2. Potential energy for the physisorption of the hydrogen molecule on a $(5,5)$ carbon nanotube. The vertical line represents the nanotube wall. The curves joining the circles, crosses, and diamonds correspond to the three approaching configurations shown in Fig. 1, from top to bottom, respectively.

We have also studied the interaction of the $\mathrm{H}_{2}$ molecule with a $(6,6)$ nanotube. The optimized radius of the nanotube is $R=7.78$ a.u. The results confirm the picture found for the interaction with the $(5,5)$ nanotube: the molecule is weakly physisorbed both outside and inside the $(6,6)$ nanotube. The depths of the external and internal wells are 0.07 and $0.12 \mathrm{eV}$, respectively. The binding energy to the external wall is nearly identical to that for the $(5,5)$ SWNT. An interesting difference is that the minimum for the molecule inside the $(6,6)$ nanotube does not occur at the position of the tube axis; it is displaced, instead, 2.5 a.u. from that axis. The difference is due to the larger radius of the $(6,6)$ nanotube. As a consequence, the depth of the inner minimum for the $(6,6)$ nanotube, i.e., $0.12 \mathrm{eV}$, is smaller than the corresponding depth, i.e., $0.17 \mathrm{eV}$, for the $(5,5)$ nanotube. Our molecular physisorption energies are consistent with those obtained by $\mathrm{Li}$ et al. [20] for $(7,7)$ nanotubes using a similar DFT method with the LDA. Their calculated value inside the $(7,7)$ nanotube is $0.11 \mathrm{eV} /$ atom. The calculated physisorption energies on the external surfaces of the nanotubes agree with the experimental value of $0.062 \mathrm{eV}$ obtained by Brown et al. [24] by thermally activated desorption of $\mathrm{H}_{2}$ from nanotubes with a radius of approximately 13 a.u. The binding energy deduced by Narehood et al. [25] from quasi-elastic neutron scattering is smaller.

From the data collected in Table I, we also notice the good agreement between the DFT and MP2 calculations for adsorption of $\mathrm{H}_{2}$ on graphene. This is noticeable since the binding energies are very small, less than $0.1 \mathrm{eV}$. Satisfactory consistency is also found between the theoretical results for graphene and nanotubes, i.e., the binding energy exhibits a dependence on the curvature of the graphitic layer. On the other hand, the theoretical calculations appear to predict higher binding energies than the measured ones. Going back to the original ques- tion of the possible enhancement of the physisorption energy due to the nanotube curvature, we notice that the enhancement is substantial for adsorption inside nanotubes, and especially for nanotubes of small radius (a similar enhancement is expected for adsorption in the small channels existing in nanotube bundles). The physisorption energy inside the $(5,5)$ nanotube, i.e., $0.17 \mathrm{eV} /$ molecule, still falls short compared to the value estimated by Li et al. [20] to be required for efficient absorption/desorption at ambient pressure and room temperature (0.15-0.2 eV/atom), although the expectations for operation under conditions of lower temperature and higher pressure look better compared to the early estimation based on the calculated physisorption energy on planar graphene.

\section{DisSOCIATIVE CHEMISORPTION}

The preferred site for the absorption of a single hydrogen atom on an SWNT is on top of a carbon atom [22], [26]-[29]; in fact, the $\mathrm{C}-\mathrm{H}$ stretching mode has been observed using Fouriertransform infrared spectroscopy [24]. We have studied the radial approach of the hydrogen atom to the nanotube following a path on top of a $\mathrm{C}$ atom. The estimated binding energies are 0.9 and $1.3 \mathrm{eV}$ for the $(5,5)$ and $(6,6)$ nanotubes, respectively. The binding energies of an $\mathrm{H}$ atom to the nanotube surface calculated previously using DFT show a wide range of values. Gülseren et al. [7], [9] and Yildirim et al. [8] predicted binding energies higher than $2 \mathrm{eV}$. Bauschlicher [26] obtained a binding energy $E_{b}=0.93 \mathrm{eV}$ for a $(10,10)$ nanotube. Lee and Lee [28] found $E_{b}=1.64 \mathrm{eV}$ for absorption on a $(5,5)$ nanotube. Our calculated binding energies fit within that range. The differences in binding energies can be ascribed to the different functionals describing exchange and correlation effects used in those works. In fact, the exchange-correlation functional has an influence even 


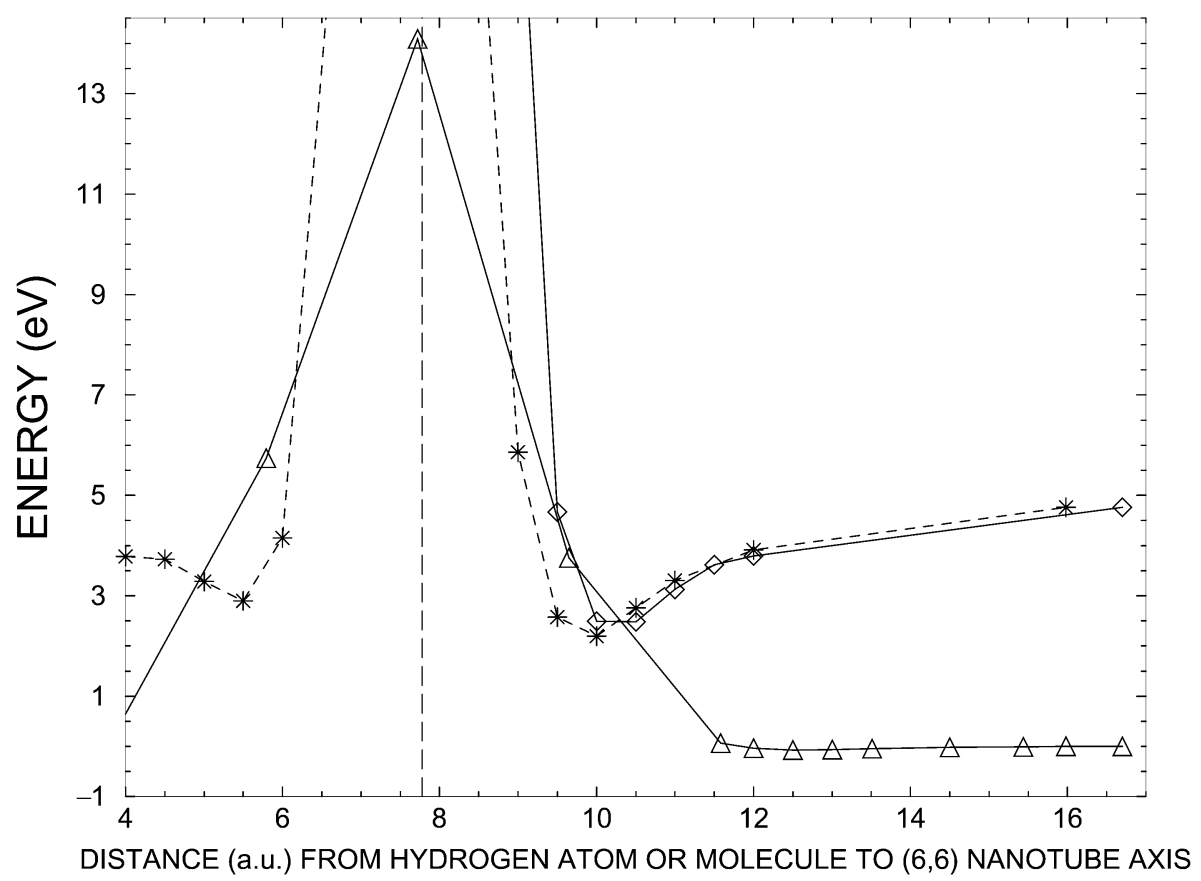

Fig. 3. Interaction energies of molecular (triangles) and atomic (diamonds and stars) hydrogen and a (6,6) SWNT. The atomic curves have been multiplied by two and shifted upwards by $4.76 \mathrm{eV}$, the dissociation energy of the $\mathrm{H}_{2}$ molecule. The vertical dashed line represents the nanotube wall.

more drastic on the molecular adsorption: the LDA gives an energy minimum for the interaction between molecular hydrogen and graphene or carbon nanotubes, while the GGA functionals produce a purely repulsive interaction [10], [11], [18].

The interaction energy as a function of the distance between the $\mathrm{H}$ atom and a $(6,6)$ SWNT is shown by the curve joining the diamonds in Fig. 3. In the calculations, the $\mathrm{C}-\mathrm{C}$ bond distances were kept frozen. Another curve, joining the stars, corresponds to the $\mathrm{H}$ atom approaching the nanotube over the midpoint of a $\mathrm{C}-\mathrm{C}$ bond. Molecular adsorption is represented by the curve joining the triangles. In order to compare atomic and molecular adsorption, the two atomic adsorption curves have been multiplied by a factor of two, and have been displaced with respect to the molecular adsorption curve by a constant shift of $4.76 \mathrm{eV}$, which is the experimental dissociation energy of the hydrogen molecule in two $\mathrm{H}$ atoms (the value calculated using the spin-polarized LDA is very close to this quantity). This figure clearly shows that two (or more) $\mathrm{H}$ atoms can be chemisorbed on the surface of the nanotube, provided that those two atoms are sufficiently far apart from each other. The $\mathrm{C}-\mathrm{H}$ bond distance is 2.5 a.u. (or 2.6 a.u. for the $(5,5)$ nanotube). However, this is not the most stable state of the system. The calculations predict, instead, that physisorption of the bound molecule on the surface of the nanotube is preferred. One can see, nevertheless, that the molecular and atomic curves cross at a distance $D_{c}=2.7$ a.u. from the nanotube wall (the nanotube wall is represented in this figure by the vertical dashed line). This means that when the molecule is forced to approach the SWNT to a distance smaller than 2.7 a.u., the molecule will dissociate. Inside the nanotube, the situation is similar. An estimation for the dissociation barrier is $2.5 \mathrm{eV}$. However, this has been obtained by introducing drastic assumptions in the calculation of the energies, namely, no relaxation of the $\mathrm{C}-\mathrm{C}$ bond lengths has been permitted when the $\mathrm{H}_{2}$ molecule, or the $\mathrm{H}$ atoms, closely approach the nanotube, and the $\mathrm{H}_{2}$ bond length was also frozen during the approaching of the molecule toward the nanotube.

Several calculations were performed, in which, for each separation between the molecule and a $(5,5)$ SWNT, the $\mathrm{H}-\mathrm{H}$ bond length was allowed to vary to minimize the total energy of the system, while still maintaining the nanotube structure frozen during the process. The orientation of the molecule in those calculations was as in configuration A of Fig. 1, i.e., with the molecular axis upon a $\mathrm{C}-\mathrm{C}$ bond. For distances between the molecule and nanotube wall higher than 3.05 a.u., relaxation of the bond length of the molecule was not observed and the picture drawn from the calculations reported in Section III is not altered. On the other hand, closer to the nanotube wall, the $\mathrm{H}-\mathrm{H}$ bond becomes stretched, its length increases, and the molecule dissociates (one can notice from Fig. 2 that, for separations $D_{a}=3$ a.u. or smaller, the molecule samples the repulsive wall of the interaction potential). At the same time, the total energy of the system decreases, but the total energy for the metastable dissociated configuration is still larger than the total energy of the molecule in the physisorption minimum so the relation between molecular physisorption and dissociative chemisorption, shown in Fig. 3 for the $(6,6)$ nanotube, remains valid.

\section{DyNAMiCAL SIMUlations}

To gain further insight into the processes that occur at close distances from the nanotube wall, we have performed damped molecular dynamics simulations, starting from several initial configurations and dynamically relaxing the system under the action of the forces on the atoms. First of all, simulations have been performed with the $\mathrm{H}_{2}$ molecule initially in the region of the physisorption well of the $(5,5)$ and $(6,6)$ nanotubes, although not at the minimum. In those cases, the dynamical evolution drives the molecule toward the physisorption minimum. 


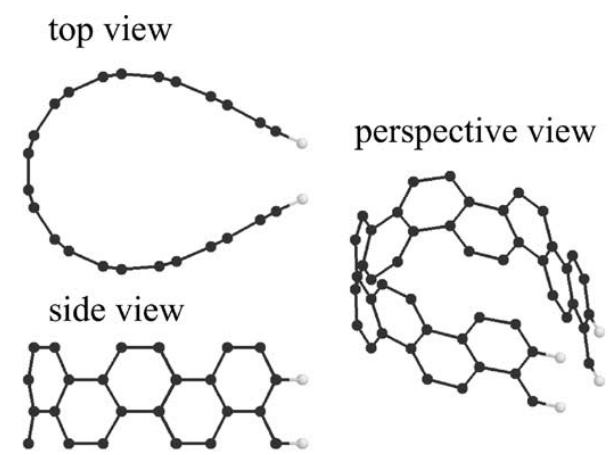

Fig. 4. Broken nanotube for a dynamical simulation starting with the dissociated hydrogen molecule outside a $(6,6)$ nanotube at a distance of 1.2 a.u. from the wall. Three views are shown.

In the final configuration, the nanotube shows no distortion, and the energy of the system is almost identical to that obtained in the static calculations discussed in Section III. The simulations also show that the axis of the molecule easily changes its orientation in the physisorption configuration.

Simulations were then performed starting with the molecule very close to the nanotube wall, at distances $D_{a}=1.1-2.1$ a.u., with the two atoms in a dissociated configuration, although relatively close to each other. This is a configuration of high potential energy, which could be achieved under high-pressure conditions, or by injecting hydrogen atoms or molecules with high kinetic energies into the reaction cell. The analysis of the atomic configurations of the system as the simulation develops in time shows that the circular cross section of the nanotubes deforms substantially due to the bonding of the two hydrogen atoms to a pair of nearest carbon atoms. Typical $\mathrm{C}-\mathrm{H}$ bond distances are 2.07-2.10 a.u., values shorter than the 2.5-2.6 a.u. found for the adsorption of an isolated $\mathrm{H}$ atom (see Section IV), and the distance between those two particular carbon atoms increases substantially. A typical snapshot for the $(6,6)$ nanotube is shown in Fig. 4. The nanotube is broken and the shortening of the $\mathrm{C}-\mathrm{H}$ bond length seems itself a consequence of the scission of the $\mathrm{C}-\mathrm{C}$ bond.

Our interpretation is that the scission of the nanotube is due to the sum of contributions from three different effects. The first one is the weakening of the $\mathrm{C}-\mathrm{C}$ bond by the concerted chemisorption of the two $\mathrm{H}$ atoms. This changes the type of bonding between each of those two carbon atoms and the environment from $s p^{2}$ to $s p^{3}$. A second important contribution is the excess potential energy initially deposited on the system, present in the configurations that we have used as the starting point of the dynamical simulations. Calculations of atomic chemisorption performed by other authors without the input of that extra potential energy do not lead to nanotube scission [26], [27], thus, we conclude that the extra potential energy has a substantial influence on the breaking of the $\mathrm{C}-\mathrm{C}$ bonds. As a final effect, it should be stressed that we have performed the simulations for a periodic superlattice geometry, with a relatively small supercell in the direction parallel to the nanotube axis. Due to the periodic repetition of the picture given in Fig. 4, there is a strip of breaking $\mathrm{C}-\mathrm{C}$ bonds along a line parallel to the nanotube axis. Scission suddenly opens up a new region in the potential energy landscape where the broken nanotube follows a steep path toward the formation of a planar graphene sheet, but this process cannot be properly handled by our simulations because the dimensions of the simulation cell in the directions perpendicular to the nanotube axis are not big enough to accommodate the complete unfolding of the nanotubes. We expect a similar breaking of $\mathrm{C}-\mathrm{C}$ bonds if a larger supercell were used in the direction parallel to the nanotube axis, but in those cases, the nanotube wall will be shattered only locally because of the lower hydrogen concentration. Those localized broken-bond defects, or the grouping of some of them, could provide a window allowing foreign atoms or molecules to go inside single-wall nanotubes through their walls, and exploring this possibility may help experimentalists to fill nanotubes with different molecules.

Our simulations have analyzed the effect of an aligned strip of dissociated $\mathrm{H}-\mathrm{H}$ pairs. Attempts to study more complex configurations than the strip would require an enlarged simulation cell. Calculations performed by Bauschlicher [26] and Bauschlicher and So [27] for nanotubes with a high coverage of atomic hydrogen predict that some particular ordered patterns are favored over random coverages. For $(10,0)$ nanotubes, a configuration of pairs of lines parallel to the nanotube axis, similar to the strip configuration, is favored with respect to others like single lines, rings, or spirals. For $(5,5)$ nanotubes, pairs of lines are again more stable than ring configurations, although a zigzag pattern related to the configuration of pairs of lines is preferred.

The weakening of $\mathrm{C}-\mathrm{C}$ bonds could help in explaining the mechanism for the coalescence of SWNTs in ropes and the doubling of SWNT diameters observed by Nikolaev et al. [30]. In those experiments, SWNT ropes were annealed in a hydrogen atmosphere at temperatures up to $1773 \mathrm{~K}$, and it was observed that some neighbor nanotubes coalesce, leading to the formation of tubes of larger diameters. According to our results, hydrogen could catalyze the coalescence of adjacent nanotubes by the weakening of $\mathrm{C}-\mathrm{C}$ bonds.

\section{CONCLUSION}

First of all, this paper has provided insight into the competition between molecular and atomic adsorption of hydrogen on carbon nanotubes. Static calculations and dynamical simulations of the interaction of molecular and atomic hydrogen with $(5,5)$ and $(6,6)$ single-wall nanotubes give the following picture: the lowest energy states of those systems are achieved for the $\mathrm{H}_{2}$ physisorbed outside and inside the nanotubes. Atomic adsorption is possible, but the dissociative chemisorption of the molecule is unfavorable since an energy barrier of approximately $2 \mathrm{eV}$ has to be overcome to dissociate the molecule on the surface of the nanotube. The second conclusion is that the calculated molecular physisorption energies are lower than those required for efficient adsorption/desorption operation at normal pressure and room temperature. The results are then not optimistic for the purposes of hydrogen storage in nanotubes or other porous graphitic materials under normal conditions of temperature and pressure, but the expectations are better for storage at lower temperatures and higher pressures. Finally, when the molecule has enough kinetic energy to closely 
approach the nanotube wall, the molecule then dissociates and the two hydrogen atoms bind strongly to two nearest neighbor carbon atoms, weakening that particular $\mathrm{C}-\mathrm{C}$ bond. The weakening of the $\mathrm{C}-\mathrm{C}$ bond, combined with an excess kinetic energy of the incoming molecule, can actually break the $\mathrm{C}-\mathrm{C}$ bond, and the self-organization of those defects along a line parallel to the nanotube axis can lead to the scission of the single-wall nanotubes.

\section{ACKNOWLEDGMENT}

The authors acknowledge the computational facilities provided by Centro de Supercomputación de Cataluña (CESCA) and the European Center for Parallelism of Barcelona (CEPBA). Author J. A. Alonso is grateful for the hospitality of Donostia International Physics Center (DIPC), San Sebastián, Spain.

\section{REFERENCES}

[1] A. C. Dillon, K. M. Jones, T. A. Bekkedahl, C. H. Kiang, D. S. Bethune, and M. J. Heben, "Storage of hydrogen in single-walled carbon nanotubes," Nature, vol. 386, pp. 377-379, 1997.

[2] A. Chambers, C. Park, R. T. K. Baker, and N. M. Rodriguez, "Hydrogen storage in graphite nanofibers," J. Phys. Chem. B, vol. 102, pp. 4253-4256, 1998

[3] P. Chen, X. Wu, J. Lin, and K. L. Tan, "High $\mathrm{H}_{2}$ uptake by alkali-doped carbon nanotubes under ambient pressure and moderate temperatures," Science, vol. 285, pp. 91-93, 1999.

[4] R. T. Yang, "Hydrogen storage by alkali-doped carbon nanotubes-revisited," Carbon, vol. 38, pp. 623-626, 2000.

[5] U. Bünger and W. Zittel, "Hydrogen storage in carbon nanostructures-Still a long road from science to commerce?," Appl. Phys. A, vol. 72, pp. 147-151, 2001

[6] V. Meregalli and M. Parrinello, "Review of theoretical calculations of hydrogen storage in carbon-based materials," Appl. Phys. A, Solid Surf., vol. 72, pp. 143-146, 2001

[7] O. Gülseren, T. Yildirim, and S. Ciraci, "Tunable adsorption on carbon nanotubes," Phys. Rev. Lett., vol. 87, p. 116802 (1-4), 2001.

[8] T. Yildirim, O. Gülseren, and S. Ciraci, "Exohydrogenated single-wall carbon nanotubes," Phys. Rev. B, Condens. Matter, vol. 64, p. 75404 (1-5), 2001.

[9] O. Gülseren, T. Yildirim, and S. Ciraci, "Effects of hydrogen adsorption on single-wall carbon nanotubes: Metallic hydrogen decoration," Phys. Rev. B, Condens. Matter, vol. 66, p. 121401 (1-4), 2002.

[10] K. Tada, S. Furuya, and K. Watanabe, "Ab initio study of hydrogen adsorption to single-walled carbon nanotubes," Phys. Rev. B, Condens. Matter, vol. 63, p. $155405(1-4), 2001$

[11] P. Dubot and P. Cenedese, "Modeling of molecular hydrogen and lithium adsorption on single-wall carbon nanotubes," Phys. Rev. B, Condens. Matter, vol. 63, p. 241402 (1-4), 2001

[12] S. P. Chan, G. Chen, X. G. Gong, and Z. F. Liu, "Chemisorption of hydrogen molecules on carbon nanotubes under high pressure," Phys. Rev Lett., vol. 87, p. 205502 (1-4), 2001

[13] M. Bockstedte, A. Kley, J. Neugebauer, and M. Scheffler, "Densityfunctional theory calculations for poly-atomic systems: Electronic structure, static and elastic properties and ab initio molecular dynamics," Comput. Phys. Commun., vol. 107, pp. 187-222, 1997.

[14] G. B. Bachelet, D. R. Hamann, and M. Schlüter, "Pseudopotentials that work: From $\mathrm{H}$ to Pu," Phys. Rev. B, Condens. Matter, vol. 26, pp. 4199-4228, 1982.

[15] D. R. Hamann, "Generalized norm-conserving pseudopotentials," Phys. Rev. B, Condens. Matter, vol. 40, pp. 2980-2987, 1989.

[16] J. S. Arellano, L. M. Molina, A. Rubio, and J. A. Alonso, "Density functional study of adsorption of molecular hydrogen on graphene layers," J. Chem. Phys., vol. 112, pp. 8114-8119, 2000.

[17] R. G. Gordon and V. S. Kim, "Theory for the forces between closed-shell atoms and molecules," J. Chem. Phys., vol. 56, pp. 3122-3133, 1972.

[18] Y. Okamoto and Y. Miyamoto, "Ab initio investigation of physisorption of molecular hydrogen on planar and curved graphenes," J. Phys. Chem. B, vol. 105, pp. 3470-3474, 2001.
[19] M. Bienfait, P. Zeppnefeld, R. C. Ramos, J. M. Gay, O. E. Vilches, and G Coddens, "Isotopic ordering in adsorbed hydrogen monolayers," Phys. Rev. B, Condens. Matter, vol. 60, pp. 11773-11782, 1999.

[20] J. Li, T. Furuta, H. Goto, T. Ohashi, Y. Fujiwara, and S. Yip, "Theoretical evaluation of hydrogen storage capacity in pure carbon nanostructures," J. Chem. Phys., vol. 119, pp. 2376-2385, 2003.

[21] L. Mattera, F. Rosatelli, C. Calvo, U. Tommasini, U. Valbusa, and G. Vidali, "Selective adsorption of ${ }^{1} \mathrm{H}_{2}$ and ${ }^{2} \mathrm{H}_{2}$ on the (0001) graphite surface," Surf. Sci., vol. 93, pp. 515-525, 1979.

[22] J. S. Arellano, L. M. Molina, A. Rubio, M. J. López, and J. A. Alonso, "Interaction of molecular and atomic hydrogen with $(5,5)$ and $(6,6)$ single-wall carbon nanotubes," J. Chem. Phys., vol. 117, pp. 2281-2288, 2002.

[23] H. Cheng, G. P. Pez, and A. C. Cooper, "Mechanism of hydrogen sorption in single-walled carbon nanotubes," J. Amer. Chem. Soc., vol. 123, pp. 5845-5846, 2001

[24] C. M. Brown, T. Yildirim, D. A. Newmann, M. J. Heben, T. Gennett, A. C. Dillon, J. L. Alleman, and J. E. Fischer, "Quantum rotation of hydrogen in single-wall carbon nanotubes," Chem. Phys. Lett., vol. 329, pp. 311-316, 2000.

[25] D. G. Narehood, J. V. Pearce, P. C. Eklund, P. E. Sokol, R. E. Lechner, J. Pieper, J. R. D. Copley, and J. C. Cook, "Diffusion of $\mathrm{H}_{2}$ adsorbed in single-walled carbon nanotubes," Phys. Rev. B, Condens. Matter, vol. 67, p. 205409 (1-5), 2003.

[26] C. W. Bauschlicher, "High coverages of hydrogen on a $(10,0)$ carbon nanotube," Nano Lett., vol. 1, pp. 223-226, 2001

[27] C. W. Bauschlicher and C. So, "High Coverages of hydrogen on $(10,0)$, $(9,0)$ and $(5,5)$ carbon nanotubes," Nano Lett., vol. 2, pp. 337-341, 2002.

[28] S. M. Lee and Y. H. Lee, "Hydrogen storage in single-walled carbon nanotubes," Appl. Phys. Lett., vol. 76, pp. 2877-2879, 2000

[29] E. C. Lee, Y. S. Kim, and K. J. Chang, "First-principles study of hydrogen adsorption on carbon nanotube surfaces," Phys. Rev. B, Condens. Matter, vol. 66, p. 073415 (1-4), 2002.

[30] P. Nikolaev, A. Thess, A. G. Rinzler, D. T. Colbert, and R. E. Smalley, "Diameter doubling of single-wall nanotubes," Chem. Phys. Lett., vol. 266, pp. 422-426, 1997.

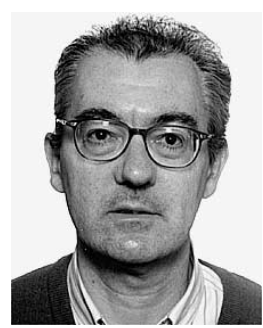

Julio A. Alonso received the Ph.D. degree in physics from the Universidad de Valladolid, Valladolid, Spain, in 1975.

From 1975 to 1980, he was a Post-Doctoral Fellow and Research Associate with the Laboratory for Research on the Structure of Matter, University of Pennsylvania, where he was involved with the theory of chemical binding in metallic alloys and also in the development of nonlocal approximations to exchange and correlation in DFT. From 1980 to 1988, he was an Associate Professor with the Universidad de Valladolid. He has been a Visiting Scientist spending sabbatical years with the University of East Anglia, Norwich, U.K., and the Donostia International Physics Center, San Sebastián, Spain, and also extended periods with the University of Osnabrück, Osnabrück, Germany, the International Center for Theoretical Physics, Trieste, Italy, and Queen's University, Kingston, ON, Canada. Since 1988, he has been with the Departamento de Física Teórica, Universidad de Valladolid, where he is currently a Professor of physics. His current research interests include DFT, amorphous alloys, atomic clusters, and carbon nanotubes.

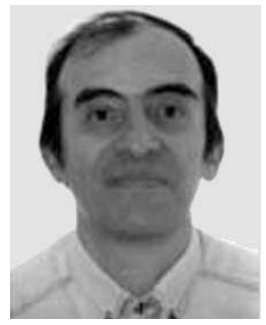

Juan S. Arellano was born in 1954. He studied physics at the National Autonomous University (UNAM), México D. F., México, and received the $\mathrm{Ph} . \mathrm{D}$. degree in physics from the UNAM, in 1987.

Since 1985, he has taught undergraduate and graduate courses for engineering students at the Universidad Autónoma Metropolitana Azcapotzalco (UAM-A), México City, México, where he has been Chairman of the physical engineering curriculum for six years. From 1999 to 2000, during a sabbatical leave with the Universidad de Valladolid, Valladolid, Spain, he became interested in the adsorption of hydrogen on graphitic materials and carbon nanotubes. His current research interests are the catalytic effect of alkali atoms to increase hydrogen absorption and the structure of alkali-intercalated graphite compounds. 


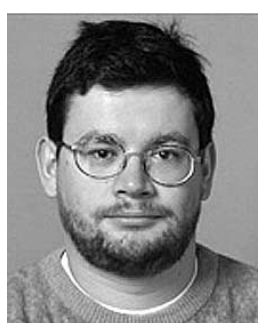

Luis M. Molina was born in 1973. He received the Ph.D. degree in physics from the Universidad de Valladolid, Valladolid, Spain, in 2001, where he was involved with cluster-assembled solids.

Since then, he has been a Post-Doctoral Research Fellow with the Institute of Physics and Astronomy, Aarhus University, Aarhus, Denmark, where he is involved with catalytic properties of gold nanoparticles. His research interests include the properties of cluster-assembled solids, catalysis on small metal particles, and carbon nanotubes.

Dr. Molina was a recipient of a 2004 Ramón y Cajal Fellowship.

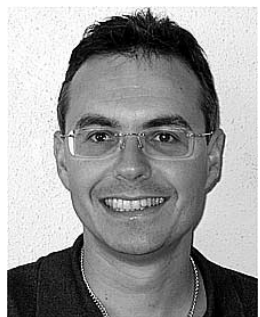

Angel Rubio was born in 1965 . He received the Ph.D. degree in physics from the Universidad de Valladolid, Valladolid, Spain, in 1991.

He is currently a Professor of condensed matter physics with the Departamento de Física de Materiales, Universidad del País Vasco, San Sebastián, Spain, and is also associated with the Donostia International Physics Center (DIPC), San Sebastián, Spain. Following a post-doctoral stay as a Fulbright Fellow with the University of California at Berkeley, in 1993, he became an Associate Professor with the Universidad de Valladolid. He has been a Visiting Scientist with the École Polytechnique, Paris, France, and the Fritz-Haber Institut, Berlin, Germany. He has edited two books on nanotechnology. His fields of interest are theoretical condensed matter physics, atomic clusters, and carbon nanotubes.

Dr. Rubio was the recipient of the Young Researchers Prize presented by the Spanish Physical Society.

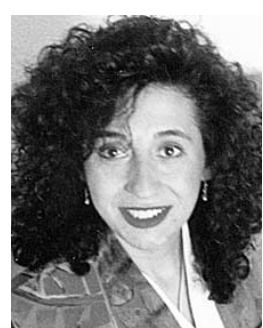

María J. López received the Ph.D. degree in physics from the Universidad de Valladolid, Valladolid, Spain, in 1990.

Since 2001, she has been a Ramón y Cajal Research Associate with the Universidad de Valladolid. From 1991 to 1993, she was a Fulbright Post-Doctoral Fellow with the Argonne National Laboratory, where she was involved with phase transitions and fragmentation of atomic clusters and the development of interatomic potentials for transition and noble metals. From 1993 to 2001, she was an Assistant Professor and Research Associate with the Universidad de Valladolid. She was a Visiting Scientist with the École Polytechnique Federal de Lausanne, Lausanne, Switzerland, and the Freie Universitat-Berlin, Berlin, Germany. Her current research focuses on nanotechnology, including atomic clusters, carbon nanotubes, and assembled materials. 